Falco Hietbrink

Marc G. H. Besselink

Willem Renooij

Luke P. H. Leenen

\section{Pitfalls in gastrointestinal permeability measurement in ICU patients}

\author{
Accepted: 12 June 2007 \\ Published online: 6 July 2007 \\ (C) Springer-Verlag 2007
}

Sir: It is hypothesized that increased intestinal permeability can induce or enhance septic complications in intensive care patients by facilitating bacterial translocation. A reliable and safe detection method would aid in identifying patients with increased intestinal permeability. Intestinal permeability has frequently been measured by tests based on the differential sugar absorption principle. In these tests the ratio of urinary recovery after orally administration of a small permeant sugar probe and large sugar probe, impermeant in the uncompromised intestine, is used as an indication of intestinal permeability [1]. The principle of these tests is that premucosal factors (i.e., gastric retention) and postmucosal factors (i.e., metabolism and renal function) are excluded because these should affect both probes similarly.
Therefore only mucosal factors (i.e., intestinal permeability) is indicated. The most commonly used test is the lactulose mannitol test (LMT). Oudemans-van Straaten et al. [2] identified confounding factors when performing the LMT in ICU patients Their study was conducted in severely ill patients with multiple organ failure. The LMT could still be applicable in patients with milder disease.

We performed the LMT on trauma patients admitted to an ICU, including all patients, with a variety of injury severity. Thirteen trauma patients were included who underwent three tests each. The trauma patients' median Injury Severity Score was 24 (range 16-38). Renal function was within normal range in all patients (creatinine $<120 \mu \mathrm{mol} / \mathrm{l}$; ureum $<7.5 \mu \mathrm{mol} / \mathrm{l})$. In 19 tests $(61 \%)$ confounding factors were identified. Of the confounding factors $53 \%$ were therapy related (i.e., mannitol use as therapeutic agent or in saline adenosine glucose mannitol). In the remaining $47 \%$ of biased tests, the factors consisted of administering problems (i.e., stomach retention).

Thus two groups of confounding factors cause a problem: (a) the use of test substances for clinical applications and (b) administering problems with the test solution. The first problem affects the probes differently, thus causing invalid test results. This can be overcome by discarding mannitol as probe. The administering problem affects the two probes similarly, and thus the ratio remains constant. In the case of gastric retention probes do not reach the intestine and cannot be measured in urine. A solution would be to administer the test fluid in the small intestine by a nasoduodenal tube.

We conclude, and stress this as a warning to future researchers, that the LMT is inappropriate not only for patients with multiple organ failure but also for trauma patients in an ICU setting (without organ failure).

\section{References}

1. Fink MP (2002) Clinical tests of gastrointestinal permeability that rely on the urinary recovery of enterally administered probes can yield invalid results in critically ill patients. Intensive Care Med 28:103-104

2. Oudemans-van Straaten HM, van der Voort PJ, Hoek FJ, Bosman RJ, van der Spoel JI, Zandstra DF (2002) Pitfalls in gastrointestinal permeability measurement in ICU patients with multiple organ failure using differential sugar absorption. Intensive Care Med 28:130-138

F. Hietbrink (®) - M. G. H. Besselink • W. Renooij - L. P. H. Leenen University Medical Center Utrecht, Surgery Department,

Heidelberglaan 100, 3508 Utrecht,

The Netherlands

e-mail: F.Hietbrink@umcutrecht.nl

Tel.: +31-30-2509882

Fax: +31-30-2541944 